\title{
Review of: "Modeling the survival times of the COVID-19 patients with a new statistical model: A case study from China"
}

Youliang chen

Potential competing interests: The author(s) declared that no potential competing interests exist.

The paper proposes an arcsine modified Weibull (ASM-Weibull) model to simulate the survival time of patients with COVID-19 epidemic, which is a very interesting work. The paper is generally well written, but in some places it will be better to improve the quality of the paper. For example, in the fifth part (the Simulation study), the simulated data set 1 and simulated data set 2 are drawn. The content is Only explain what each figure represents, without analyzing the results. 